\title{
Plantas usadas en afecciones digestivas en Paraguay
}

\author{
Plants used in digestive diseases in Paraguay
}

\author{
Nélida Soria Rey ${ }^{1,2 *}$ (10 \\ ${ }^{1}$ Universidad Nacional de Pilar, Facultad de Ciencias Aplicadas. Pilar, \\ Paraguay. \\ ${ }^{2}$ Sociedad Científica del Paraguay, Asunción, Paraguay.
}

Autor correspondiente: nsoria2000@yahoo.com

Resumen: La OMS promueve el uso de la Medicina Tradicional en la Atención Primaria de Salud debido a que ésta ha demostrado ser eficaz como tratamiento y prevención de primera línea para enfermedades agudas, tales como resfriados, diarreas, dolores de estómago, fiebres ligeras, entre otras, especialmente en sus fases iniciales. Este trabajo forma parte de la actualización de plantas medicinales que se realiza cada año, para ello se realizaron muestreos en los mercados de Asunción, San Lorenzo, Caaguazú, Villarrica, Pilar, durante todo el año 2019. En cada una de las comunidades estudiadas, se realizaron 14 entrevistas semi estructuradas a los vendedores y usuarios de plantas medicinales que asistían a consultas en las Unidades de Salud Familiar. Se identificaron las especies empleadas por los usuarios para las afecciones del sistema digestivo. 70 especies se emplean para uso digestivo. La familia mejor representada fue la Asteraceae con 11 especies y 9 géneros. El órgano vegetal más utilizado fue la hoja. Se calculó la importancia relativa de las enfermedades, encontrándose que el dolor de estómago es la afección más frecuentemente mencionada y las tres especies citadas con mayor frecuencia para el uso como antiespasmódico estomacal fueron Mentha x piperita "menta í", Lippia alba "salvia rã", Mangifera indica "mango". Los estudios científicos confirmaron que dos de las especies mencionadas en este grupo presentaron acción similar a la mencionada en los conocimientos tradicionales.

Palabras clave: etnobotánica, afecciones digestivas, plantas medicinales.

Abstract: The WHO promotes the use of Traditional Medicine in Primary Health Care because it has proven to be effective as a first-line treatment and prevention for acute illnesses, such as colds, diarrhea, stomach aches, 
mild fevers, among others. especially in its early stages. This work is part of the update of medicinal plants that is carried out each year, for this, samples were carried out in the markets of Asunción, San Lorenzo, Caaguazú, Villarrica, Pilar, throughout 2019. 14 semi-structured interviews were conducted with the sellers and users of medicinal plants and who attended consultations in the Family Health Units, in each of the communities. 70 species were identified, used for their digestive use the best represented family was the Asteraceae with 11 species and 9 genera. The most used plant organ was the leaf. When the relative importance of diseases is analyzed, we find that stomach pain is considered the most important condition and the three species most frequently cited for use as stomach antispasmodic were Mentha x piperita "menta í", Lippia alba "salvia rã ", Mangifera indicates" mango ". It also mentions some species that have demonstrated efficacy in their action similar to that mentioned in traditional knowledge.

Keywords: ethnobotany, digestive disorders, medicinal plants.

\section{INTRODUCCIÓN}

La OMS promueve el uso de la Medicina Tradicional en la Atención Primaria de Salud (APS) debido a que ésta ha demostrado ser eficaz como tratamiento y prevención de primera línea para enfermedades agudas, tales como resfriados, diarreas, dolores de estómago, fiebres ligeras, entre otras, especialmente en sus fases iníciales. En segundo lugar, esta forma de medicina representa en muchos países, y en ciertos casos la única forma de atención asequible, accesible, y disponible. Se estima que alrededor del 80\% de la población mundial recurre a la medicina tradicional herbolaria para la APS. Además, la incorporación de esta medicina reduciría costos, ya que experiencias en muchos países del mundo demuestran que con el uso eficiente de las plantas medicinales en APS, se logró reducir hasta el 20\% de los gastos en Salud Pública. Las afecciones digestivas se encuentran entre las que pueden tratarse con plantas, y suponen aproximadamente el $20 \%$ de las consultas de atención primaria(1-2).

En el contexto latinoamericano, las experiencias aplicadas desde un enfoque intercultural, se han caracterizado generalmente por un tratamiento a la salud desconectado de los problemas de las poblaciones y comunidades, sin considerar el grado de confianza en las especies, sin vínculo con la estructura social, económica, lo que da como resultado una medicina ancestral cada vez más separada del sistema de salud(3-4). Esto se vuelve muy real debido al intercambio cultural ya que en los últimos tiempos las 
fronteras prácticamente han desaparecido y la migración contribuye a los encuentros culturales ocasionando la declinación del conocimiento tradicional y de la identidad propia ${ }^{(5)}$.

En Paraguay, el uso de plantas medicinales es frecuente para tratar diversas afecciones o como medida preventiva. Podemos afirmar que casi el $90 \%$ de los habitantes de Paraguay las emplea con alguna asiduidad, aunque no se encuentren incorporadas al sistema público de salud y son poco utilizadas en la medicina formal, por lo que su prescripción es prácticamente inexistente en los servicios de salud. Es poco frecuente que los médicos acepten el uso de las plantas para tratar enfermedades y que recomienden su empleo, aun cuando éste se encuentre respaldado por la evidencia científica( ${ }^{(6)}$.

En este trabajo se presentan las especies medicinales utilizadas en Paraguay para las afecciones digestivas, algunas de las cuales han demostrado efectividad y podrían emplearse en la atención primaria de salud, considerando los resultados científicos que puedan avalar el uso seguro y eficaz de las especies en la atención primaria de salud.

\section{METODOLOGÍA}

Este trabajo forma parte de la actualización de plantas medicinales. Se realizaron muestreos en los mercados de Asunción, San Lorenzo, Caaguazú, Villarrica, Pilar, durante todo el año 2019. Se realizaron 14 entrevistas semi estructuradas a los vendedores y usuarios de plantas medicinales y que asistían a consultas en las Unidades de Salud Familiar, en cada una de las comunidades. Los entrevistados accedieron a participar de la encuesta, proporcionando la información que le fuera requerida. En algunas ocasiones se realizó la recolección de las especies para su correcta identificación botánica, acompañado de los vendedores y/o usuarios. Las muestras fueron herborizadas, etiquetadas, secadas y procesadas para su conservación. Estas muestras fueron identificadas taxonómicamente utilizando las técnicas tradicionales de comparación empleando claves taxonómicas, bibliografía disponible y base de datos accesibles en internet(7-10), conservando el testigo patrón en el Museo de Etnobotánica, de la Universidad Nacional de Caaguazú (UNCA) y en la Sociedad Científica del Paraguay. Las afecciones mencionadas por los informantes se clasificaron utilizando la Clasificación Estadística Internacional de Enfermedades y Problemas Relacionados con la Salud (CIE-10) realizada por OPS(11). Además, se realizó una revisión de la bibliografía para la validación científica del uso de las mismas. Para 
determinar La importancia de las enfermedades mencionadas por los informantes, se anotó el número de veces que la afección fue citada por los informantes (Número Absoluto de Citas, NAC) y el número de especies utilizadas para su atención (Número Absoluto de Especies, NAE). Se calculó el Número Relativo de Citas (NRC) dividiendo el número de citas que recibe una enfermedad entre el número total de informantes (70) y multiplicando el resultado por 100. También se obtuvo el Número Relativo de Especies (NRE) dividiendo el número de especies utilizadas para la atención de una enfermedad entre el total de especies registradas (73) y multiplicando el resultado por 100. Con estos valores se calculó el Índice de Importancia Relativa de las Enfermedades (IIRE) utilizando la formula modificada por Ortega-Cala et al.(12): IIRE = NCR + NRE. Los valores más altos se obtienen para las enfermedades que son mencionadas por un mayor número de informantes y para las que se utiliza un mayor número de especies de plantas medicinales.

\section{RESULTADOS}

Los resultados etnobotánicos muestran que se identificaron 70 especies, empleadas para su uso digestivo. La familia mejor representada fue Asteraceae con 11 especies y 9 géneros (15\%), seguido de Verbenaceae 8 especies 3 géneros (7\%) y Lamiaceae 4 especies 3 géneros $(6,8 \%)$. El órgano vegetal más utilizado fue la hoja, seguido de la parte aérea, planta entera (Figura 1).

Las 70 especies medicinales se encuentran vinculadas a 11 afecciones del aparato digestivo (Tabla 1). En la Tabla 2 se citan las especies reportadas para su uso en las afecciones del aparato digestivo y se menciona la importancia relativa de las enfermedades (IIRE) según los informantes. Los resultados demuestran que el dolor de estómago es la afección con mayor importancia relativa, seguido de los problemas de digestión y del estreñimiento.

Al analizar la importancia relativa de las enfermedades, encontramos que el dolor de estómago está considerado como la afección más importante y las tres especies citadas con mayor frecuencia para el uso como antiespasmódico estomacal fueron Mentha x piperita "menta í", Lippia alba "salvia rã", Mangifera indica "mango". Como digestivo se citaron a Eryngium elegans "turu tu'i", Artemisia absinthium "ajenjo", Baccharis trimera "Yaguarete Kaa". En la Tabla 2 se muestra las especies, mencionadas por los usuarios, empleadas para las afecciones del sistema digestivo. 
Rev. Soc. cient. Parag. 2021;26(2):163-176

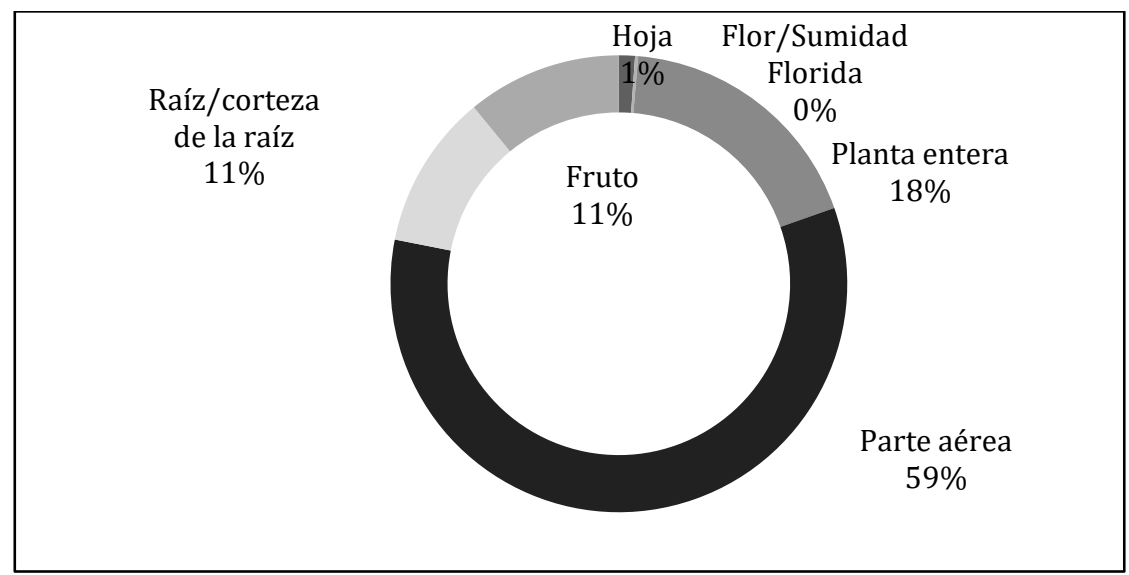

Figura 1. Órganos vegetales más utilizados.

Fuente. Elaboración propia.

Tabla 1. Importancia relativa de las afecciones digestivas

\begin{tabular}{|c|c|c|c|c|c|}
\hline Afección & $\begin{array}{l}\text { Número de } \\
\text { citas por } \\
\text { informante } \\
\text { (NAC) }\end{array}$ & $\begin{array}{l}\text { Número } \\
\text { relativo } \\
\text { de Citas } \\
\text { (NCR) } \\
\end{array}$ & $\begin{array}{l}\text { Número } \\
\text { de } \\
\text { especies } \\
\text { (NAE) } \\
\end{array}$ & $\begin{array}{l}\text { Número } \\
\text { Relativo } \\
\text { de } \\
\text { Especies }\end{array}$ & $\begin{array}{l}\text { Importancia } \\
\text { relativa de las } \\
\text { enfermedades } \\
\text { IIRE }\end{array}$ \\
\hline Diarrea & 6 & 8,57 & 4 & 5,47 & 14 \\
\hline Disenterías & 3 & 4,28 & 1 & 1,36 & 5,64 \\
\hline $\begin{array}{l}\text { Dolor de } \\
\text { estomago }\end{array}$ & 50 & 43 & 30 & 41 & 84 \\
\hline Digestivo & 21 & 30 & 12 & 16,43 & 46,43 \\
\hline Laxante & 4 & 5,71 & 2 & 13,30 & 19,1 \\
\hline Purgante & 4 & 5,71 & 2 & 13,30 & 19,1 \\
\hline Gastritis & 3 & 4,28 & 2 & 2,73 & 7,01 \\
\hline $\begin{array}{l}\text { Empacho } \\
\text { de niños }\end{array}$ & 7 & 10 & 2 & 2,73 & 12,73 \\
\hline $\begin{array}{l}\text { Ulceras } \\
\text { gástricas }\end{array}$ & 3 & 4,10 & 2 & 2,73 & 6,83 \\
\hline Vómitos & 2 & 2,85 & 2 & 2,73 & 5,58 \\
\hline $\begin{array}{l}\text { Tónico } \\
\text { amargo }\end{array}$ & 3 & 4,28 & 1 & 1,38 & 5,66 \\
\hline
\end{tabular}

Fuente: Elaboración propia 
Rev. Soc. cient. Parag. 2021;26(2):163-176

Tabla 2. Especies medicinales usadas en afecciones digestivas

\begin{tabular}{|c|c|c|c|c|c|}
\hline Familia & $\begin{array}{l}\text { Nombre } \\
\text { científico }\end{array}$ & $\begin{array}{l}\text { Nombre } \\
\text { popular }\end{array}$ & Usos & Órg. Utili. & $\begin{array}{l}\mathrm{Ci} \\
\text { ta }\end{array}$ \\
\hline Adoxaceae & $\begin{array}{l}\text { Sambucus } \\
\text { autralis } \\
\text { Cham. \& } \\
\text { Schltdl. } \\
\end{array}$ & Sauco & $\begin{array}{l}\text { Antiespasmódi } \\
\text { co estomacal }\end{array}$ & Hoja & 4 \\
\hline $\begin{array}{l}\text { Amaranthacea } \\
\text { e }\end{array}$ & $\begin{array}{l}\text { Alternanther } \\
\text { a pungens } \\
\text { H.B.K } \\
\end{array}$ & $\begin{array}{l}\text { Perdudilla } \\
\text { negra }\end{array}$ & $\begin{array}{l}\text { Hepatitis, } \\
\text { antidiarreico }\end{array}$ & Parte aérea & 5 \\
\hline $\begin{array}{l}\text { Amaranthacea } \\
\text { e }\end{array}$ & $\begin{array}{l}\text { Gomphrena } \\
\text { perennis L. }\end{array}$ & Siempre vive & $\begin{array}{l}\text { Afecciones } \\
\text { cardíacasTran } \\
\text { quilizante }\end{array}$ & $\begin{array}{l}\text { Sumidad } \\
\text { florida/fructifi } \\
\text { cada }\end{array}$ & 1 \\
\hline Anacardiaceae & $\begin{array}{l}\text { Mangifera } \\
\text { indica } \mathrm{L} .\end{array}$ & Hoja de mango & $\begin{array}{l}\text { Antiespasmódi } \\
\text { co estomacal }\end{array}$ & Hoja & 12 \\
\hline Apiaceae & $\begin{array}{l}\text { Pimpinella } \\
\text { anisum L. }\end{array}$ & Anis & $\begin{array}{l}\text { Antiespasmódi } \\
\text { co estomacal }\end{array}$ & Fruto & 2 \\
\hline Apiaceae & $\begin{array}{l}\text { Anethum } \\
\text { graveolens L. }\end{array}$ & Eneldo & $\begin{array}{l}\text { Digestivo, } \\
\text { galactogeno }\end{array}$ & Fruto & 2 \\
\hline Apiaceae & $\begin{array}{l}\text { Foeniculum } \\
\text { vulgare Mill. }\end{array}$ & Hinojo & $\begin{array}{l}\text { Antiespasmódi } \\
\text { co estomacal }\end{array}$ & Parte aérea & 4 \\
\hline Apiacea & $\begin{array}{l}\text { Eryngium } \\
\text { elegans Cham } \\
\text { \& Schtdl. }\end{array}$ & Turu tu'i & $\begin{array}{l}\text { Digestivo, } \\
\text { hepático. }\end{array}$ & Hoja & 5 \\
\hline Apocymaceae & $\begin{array}{l}\text { Aspidosperm } \\
\text { a quebracho- } \\
\text { blanco } \\
\text { Schlecht. }\end{array}$ & $\begin{array}{l}\text { Quebracho } \\
\text { blanco }\end{array}$ & $\begin{array}{l}\text { Digestivo, } \\
\text { antipirético. } \\
\text { Uso externo } \\
\text { para lavado de } \\
\text { heridas }\end{array}$ & Hoja & 3 \\
\hline Asteraceae & $\begin{array}{l}\text { Achyrocline } \\
\text { alata (Kunth) } \\
\text { DC. }\end{array}$ & $\begin{array}{l}\text { Marcela } \\
\text { Cocuere }\end{array}$ & $\begin{array}{l}\text { Antiespasmódi } \\
\text { co estomacal }\end{array}$ & Parte aérea & 1 \\
\hline
\end{tabular}




\begin{tabular}{|c|c|c|c|c|c|}
\hline Asteraceae & $\begin{array}{l}\text { Artemisia } \\
\text { absinthium L. }\end{array}$ & Ajenjo & $\begin{array}{l}\text { Digestivo, } \\
\text { empacho de } \\
\text { niños, } \\
\text { antidiabético, } \\
\text { abortivo, } \\
\text { emenagogo y } \\
\text { para combatir } \\
\text { la malaria. }\end{array}$ & Hoja & 5 \\
\hline Asteraceae & $\begin{array}{l}\text { Baccharis } \\
\text { articulata } \\
\text { (Lam.) Pers. }\end{array}$ & Chirca Melosa & $\begin{array}{l}\text { Antiespasmódi } \\
\text { co estomacal }\end{array}$ & Parte aérea & 2 \\
\hline Asteraceae & $\begin{array}{l}\text { Baccharis } \\
\text { trimera Less. }\end{array}$ & Yaguarete Kaa & $\begin{array}{l}\text { Digestivo, } \\
\text { tónico amargo }\end{array}$ & Parte aérea & \\
\hline Asteraceae & $\begin{array}{l}\text { Carthamus } \\
\text { tinctorius L. }\end{array}$ & Asafran & Digestivo & Flor & 2 \\
\hline Asteraceae & $\begin{array}{l}\text { Austroeupato } \\
\text { rium } \\
\text { inulifolium } \\
\text { (Kunth) R.M. } \\
\text { King \& H. Rob. } \\
\end{array}$ & Doctorcito & Antidiarreico & $\begin{array}{l}\text { Sumidad } \\
\text { florida/fructifi } \\
\text { cada }\end{array}$ & 1 \\
\hline Asteraceae & $\begin{array}{l}\text { Campuloclinu } \\
\text { m } \\
\text { macrocephal } \\
\text { um (Less.) DC. }\end{array}$ & Teju Ka’a & $\begin{array}{l}\text { Antiespasmódi } \\
\text { co estomacal }\end{array}$ & Parte aérea & 1 \\
\hline Asteraceae & $\begin{array}{l}\text { Hypochoeris } \\
\text { microcephala } \\
\text { (SCh. Bip) } \\
\text { Cabrera }\end{array}$ & Achicoria & Digestivo & Planta entera & 1 \\
\hline Asteraceae & $\begin{array}{l}\text { Pluchea } \\
\text { sagittalis } \\
\text { Lam. }\end{array}$ & $\begin{array}{l}\text { Yerba de } \\
\text { lucero }\end{array}$ & $\begin{array}{l}\text { Para producir } \\
\text { el vómito en } \\
\text { casos de } \\
\text { empacho en } \\
\text { niños, } \\
\text { Antidiarreico }\end{array}$ & Parte aérea & 1 \\
\hline Asteraceae & $\begin{array}{l}\text { Porophyllum } \\
\text { ruderale } \\
\text { (Jacq.) Cass. }\end{array}$ & Yryvu Canilla & $\begin{array}{l}\text { Antiespasmódi } \\
\text { co estomacal }\end{array}$ & Parte aérea & 4 \\
\hline Asteraceae & $\begin{array}{l}\text { Tagetes } \\
\text { minuta } \mathrm{L} .\end{array}$ & Suico & $\begin{array}{l}\text { Antiespasmódi } \\
\text { co estomacal }\end{array}$ & Parte aérea & 9 \\
\hline
\end{tabular}


Rev. Soc. cient. Parag. 2021;26(2):163-176

\begin{tabular}{llllll}
\hline Cactaceae & $\begin{array}{l}\text { Pereskia } \\
\text { nemorosa } \\
\text { Rojas Acosta }\end{array}$ & Amapola & $\begin{array}{l}\text { Antiespasmódi } \\
\text { co estomacal }\end{array}$ & Hoja, Flor & 1 \\
\hline Celastraceae & $\begin{array}{l}\text { Maytenus } \\
\text { ilicifolia } \text { Mart. }\end{array}$ & Cangorosa & $\begin{array}{l}\text { dispepsia, } \\
\text { pirosis y la } \\
\text { gastritis }\end{array}$ & $\begin{array}{l}\text { Hoja, corteza } \\
\text { de la raíz }\end{array}$ & 2 \\
\hline
\end{tabular}

\begin{tabular}{|c|c|c|c|c|}
\hline Costaceae & $\begin{array}{l}\text { Costus } \\
\text { arabigus L }\end{array}$ & Caña brava & $\begin{array}{l}\text { Diurético, } \\
\text { Antiespasmodi } \\
\text { co estomacal }\end{array}$ & $\begin{array}{l}\text { Hojas, } \\
\text { Rizomas }\end{array}$ \\
\hline Euphorbiaceae & Croton sp. & $\begin{array}{l}\text { Mister del } \\
\text { Campo }\end{array}$ & $\begin{array}{l}\text { Antiespasmódi } \\
\text { co estomacal }\end{array}$ & Parte aérea \\
\hline
\end{tabular}

$\begin{array}{llll} & \text { Bauhinia } & & \text { Carminativo, } \\ \text { Fabaceae } & \text { Pandicans } & \text { Pata de buey } & \text { en digestiones } \\ \text { Benth. } & & \text { lentas, } \\ & \text { flatulencias. }\end{array}$

\begin{tabular}{|c|c|c|c|c|c|}
\hline Fabaceae & Senna sp. & Pito Yva & $\begin{array}{l}\text { Antiespasmódi } \\
\text { co estomacal }\end{array}$ & Hoja & 1 \\
\hline Lamiaceae & Lavandula sp. & Alucema & $\begin{array}{l}\text { Antiespasmódi } \\
\text { co estomacal }\end{array}$ & Parte aérea & 2 \\
\hline Lamiaceae & $\begin{array}{l}\text { Plectranthus } \\
\text { amboinicus } \\
\text { (Lour.) } \\
\text { Spreng. }\end{array}$ & Boldo guasu & $\begin{array}{l}\text { Antiespasmódi } \\
\text { co estomacal }\end{array}$ & Hoja & 10 \\
\hline \multirow[t]{2}{*}{ Lamiaceae } & \multirow[t]{2}{*}{ Mentha sp. } & \multirow[t]{2}{*}{ Menta } & $\begin{array}{l}\text { Antiespasmódi } \\
\text { co estomacal, } \\
\text { Taquicardia. }\end{array}$ & \multirow[t]{2}{*}{ Hoja } & \multirow[t]{2}{*}{1} \\
\hline & & & Tranquilizante & & \\
\hline \multirow[t]{2}{*}{ Lamiaceae } & \multirow[t]{2}{*}{$\begin{array}{l}\text { Mentha } x \\
\text { piperita } \mathrm{L} .\end{array}$} & \multirow[t]{2}{*}{ Menta í } & $\begin{array}{l}\text { Antiespasmódi } \\
\text { co estomacal, } \\
\text { Taquicardia }\end{array}$ & \multirow[t]{2}{*}{ Hoja } & \multirow[t]{2}{*}{27} \\
\hline & & & Tranquilizante & & \\
\hline
\end{tabular}


Rev. Soc. cient. Parag. 2021;26(2):163-176

\begin{tabular}{|c|c|c|c|c|c|}
\hline Lythraceae & $\begin{array}{l}\text { Cuphea } \\
\text { racemosa } \\
\text { (Lf.) Spreng. }\end{array}$ & $\begin{array}{l}\text { Perchicaria, } \\
\text { siete sangria }\end{array}$ & Antidiarreico & Parte aérea & \\
\hline Monimiaceae & $\begin{array}{l}\text { Peumus } \\
\text { boldus } \\
\text { Molina }\end{array}$ & Boldo & $\begin{array}{l}\text { Colagogo, } \\
\text { carminativo }\end{array}$ & Fruto & 10 \\
\hline Moraceae & $\begin{array}{l}\text { Dorstenia } \\
\text { brasiliensis } \\
\text { Lam. } \\
\end{array}$ & Tarope & $\begin{array}{l}\text { Antiespasmódi } \\
\text { co estomacal, } \\
\text { Antifebril }\end{array}$ & Planta entera & 13 \\
\hline Myrtaceae & $\begin{array}{l}\text { Campomanesi } \\
\text { a pubescens } \\
\text { (AP de C.) } \\
\text { Berg }\end{array}$ & Guavirami & Antidiarreico & Hoja & 1 \\
\hline Myrtaceae & $\begin{array}{l}\text { Psidium } \\
\text { guajava L. }\end{array}$ & Guayaba & $\begin{array}{l}\text { Antidiarreico, } \\
\text { en disenterías }\end{array}$ & Hoja & 3 \\
\hline $\begin{array}{l}\text { Phytolaccacea } \\
\text { e }\end{array}$ & $\begin{array}{l}\text { Petiveria } \\
\text { alliaceae } \mathrm{L} .\end{array}$ & Pipi rapo & $\begin{array}{l}\text { Antiespasmódi } \\
\text { co estomacal }\end{array}$ & $\begin{array}{l}\text { corteza de la } \\
\text { raíz }\end{array}$ & 1 \\
\hline Piperaceae & $\begin{array}{l}\text { Piper } \\
\text { regnellii } \\
\text { (Miq.) C. DC. }\end{array}$ & Jagua Rundi & Antitusivo & Hoja & 1 \\
\hline Piperaceae & Peperomia sp. & Jatevu Ka'a & $\begin{array}{l}\text { Antiespasmódi } \\
\text { co estomacal }\end{array}$ & Planta entera & 1 \\
\hline Plantaginaceae & $\begin{array}{l}\text { Plantago } \\
\text { tomentosa } \\
\text { Lam. }\end{array}$ & $\begin{array}{l}\text { Llantén de } \\
\text { tierra }\end{array}$ & $\begin{array}{l}\text { Antiespasmódi } \\
\text { co estomacal }\end{array}$ & Planta entera & 2 \\
\hline Polygonaceae & $\begin{array}{l}\text { Polygonum } \\
\text { puntatum } \\
\text { Elliot } \\
\end{array}$ & Kaata'i & $\begin{array}{l}\text { Vermicida, } \\
\text { parásitos } \\
\text { internos }\end{array}$ & Parte aérea & 1 \\
\hline Poaceae & $\begin{array}{l}\text { Cymbopogon } \\
\text { Citratus L. }\end{array}$ & Cedron Kapi'i & $\begin{array}{l}\text { cólicos } \\
\text { intestinales, } \\
\text { úlceras } \\
\text { gastricas } \\
\end{array}$ & Hoja & \\
\hline Rosaceae & $\begin{array}{l}\text { Eriobotrya } \\
\text { japonica } \\
\text { (Thunb.) Lindl. }\end{array}$ & Nispero & $\begin{array}{l}\text { Diurético, } \\
\text { Antiespasmodi } \\
\text { co estomacal }\end{array}$ & Hoja & 2 \\
\hline Rosaceae & Rosa sp. & $\begin{array}{l}\text { Rosa } \\
\text { Mosqueta }\end{array}$ & Laxante & Flor & 1 \\
\hline Rubiaceae & $\begin{array}{l}\text { Genipa } \\
\text { americana L. }\end{array}$ & Ñandypa & Purgante & Raíz & \\
\hline
\end{tabular}


Rev. Soc. cient. Parag. 2021;26(2):163-176

\begin{tabular}{|c|c|c|c|c|c|}
\hline Rutaceae & Citrus sp. & Naranja Hai & $\begin{array}{l}\text { Tranquilizante } \\
\text { Antiespasmodi } \\
\text { co estomacal }\end{array}$ & Hoja & 2 \\
\hline Salicaceae & $\begin{array}{l}\text { Casearia } \\
\text { sylvestris SW. }\end{array}$ & Burro Ka'a & $\begin{array}{l}\text { Antiespasmódi } \\
\text { co estomacal }\end{array}$ & Hoja & 1 \\
\hline Santalaceae & $\begin{array}{l}\text { Phoradendro } \\
\boldsymbol{n} \text { sp. }\end{array}$ & Ka'avoty ry & $\begin{array}{l}\text { Antiespasmódi } \\
\text { co estomacal }\end{array}$ & Planta entera & 1 \\
\hline $\begin{array}{l}\text { Scrophulariace } \\
\text { ae }\end{array}$ & $\begin{array}{l}\text { Scoparia } \\
\text { dulcis } \mathrm{L} .\end{array}$ & $\begin{array}{l}\text { Typycha } \\
\text { kuratu }\end{array}$ & Digestivo & & 3 \\
\hline Ulmaceae & Celtis sp. & Yoasy'y & $\begin{array}{l}\text { Antidiarreico, } \\
\text { Antitusígeno }\end{array}$ & Hoja & 1 \\
\hline Verbenaceae & $\begin{array}{l}\text { Aloysia } \\
\text { polystachya } \\
\text { Griseb. \& } \\
\text { Moldenke }\end{array}$ & Burrito & $\begin{array}{l}\text { Antiespasmódi } \\
\text { co estomacal }\end{array}$ & Hoja & 35 \\
\hline Verbenaceae & $\begin{array}{l}\text { Lippia } \\
\text { brasiliensis } \\
\text { (Link.) T. Silva } \\
\end{array}$ & $\begin{array}{l}\text { Jatei Ka a } \\
\text { Ka'aguy }\end{array}$ & $\begin{array}{l}\text { Antiespasmódi } \\
\text { co estomacal }\end{array}$ & Hoja & 1 \\
\hline Verbenaceae & Lippia sp. & Poleo & $\begin{array}{l}\text { Digestivo, } \\
\text { Antiespasmodi } \\
\text { co estomacal }\end{array}$ & Hoja & 1 \\
\hline Verbenaceae & $\begin{array}{l}\text { Aloysia } \\
\text { gratissima } \\
\text { (Gillies \& } \\
\text { Hook.) Tronc }\end{array}$ & Poleo'i & $\begin{array}{l}\text { Digestivo, } \\
\text { Antiespasmodi } \\
\text { co estomacal }\end{array}$ & Hoja & 3 \\
\hline Verbenaceae & $\begin{array}{l}\text { Aloysia } \\
\text { triphylla } \\
\text { Royle. } \\
\end{array}$ & $\begin{array}{l}\text { Cedrón } \\
\text { Paraguay o } \\
\text { Yerba Luisa } \\
\end{array}$ & $\begin{array}{l}\text { digestivo y } \\
\text { antiespasmódi } \\
\text { co. }\end{array}$ & Hoja & \\
\hline Verbenaceae & $\begin{array}{l}\text { Lippia alba } \\
\text { (Mill.) N.E. Br. } \\
\text { ex Britton \& P. } \\
\text { Wilson }\end{array}$ & Salvia rã & $\begin{array}{l}\text { Antiespasmódi } \\
\text { co estomacal }\end{array}$ & Hoja & 28 \\
\hline Verbenaceae & $\begin{array}{l}\text { Verbena } \\
\text { bonariensis } \mathrm{L} . \\
\text { var. } \\
\underline{\text { bonariensis }}\end{array}$ & Verbena & $\begin{array}{l}\text { Antiespasmódi } \\
\text { co estomacal }\end{array}$ & Parte aérea & 5 \\
\hline
\end{tabular}


Rev. Soc. cient. Parag. 2021;26(2):163-176

\begin{tabular}{llllll}
\hline Verbenaceae & $\begin{array}{l}\text { Verbena graci } \\
\text { lescens (Cham } \\
\text {.) Herter }\end{array}$ & Verbena 'i & $\begin{array}{l}\text { Antiespasmódi } \\
\text { co estomacal }\end{array}$ & Parte aérea & 5 \\
\hline $\begin{array}{l}\text { Xanthorrhoeac } \\
\text { eae }\end{array}$ & $\begin{array}{c}\text { Aloe vera (L.) } \\
\text { Burm. f. }\end{array}$ & Aloe pititi & $\begin{array}{l}\text { Laxante, } \\
\text { purgante }\end{array}$ & Hoja & 3 \\
\hline
\end{tabular}

\section{DISCUSIÓN}

La Familia medicinal predominante varia de localidad en localidad, en Cuba, por ejemplo, Fabaceae es la familia mejor representada, mientras que en Ecuador es la Asteraceae, coincidiendo con los resultados obtenidos en este trabajo. El órgano vegetal más empleado es la hoja coincidiendo con lo mencionado para Cuba, Ecuador(13-14).

El dolor de estómago fue la afección del sistema digestivo mencionado con mayor frecuencia y para las que se utilizaron mayor cantidad de especies vegetales. Estos resultados son similares a los mencionados para Ecuador donde el número de especies empleadas para las afecciones gastro intestinales y las asociadas al sistema respiratorio son las más numerosas ${ }^{(13) .}$

Se menciona la eficacia demostrada de cinco especies con actividad en pruebas farmacologicas y/o clínicas realizadas, coincidiendo con el uso popular.

a) Mentha $x$ piperita cuya actividad ha sido evaluada exitosamente contra parásitos como Schistosoma mansoni y Giardia lamblia(15-16) y además, ha demostrado una acción antibiótica en el tratamiento de Helicobacter pylori(17).

b) El extracto acuoso de L. alba plantea la potencial utilidad terapéutica de la planta en personas con diferentes formas de gastritis y con úlceras pépticas aprovechando el efecto gastroprotector e inhibidor de la planta sobre H. pylori(18).

c) Así también se realizaron algunos estudios clínicos como por ejemplo el realizado con la tintura de Psidum guajava que preparada al $20 \%$ de hoja equivalente a $17 \mathrm{mg}$ de flavonoides, disuelto en un poco de agua y administrado a los pacientes cada 8 hs, por tres días, tuvo un efecto antidiarreico importante ya que las diarreas desaparecieron(19). 
d) Artemisia absinthium que ha sido evaluada exitosamente en el tratamiento de la gastritis y las ulceras producidas por Helycobacter pilori(20-21).

e) Scoparia dulcis especie mencionada por los informantes como digestivo, presenta estudios que demuestran que la especie posee efecto antiespasmódico en cólicos estomacales(22).

\section{CONCLUSIÓN}

Como se ve existe coincidencia del uso atribuido a las especies en la comunidad con los estudios científicos, esto es una evidencia de la efectividad del uso médico tradicional asignado a las plantas y por lo tanto, de su importancia para la atención de la salud de quienes las utilizan como parte de su tradición. Es necesario continuar con la recolección de datos etnobotánicos y su validación a través de los estudios químicos llegando a los clínicos.

\section{REFERENCIAS BIBLIOGRÁFICAS}

1. OMS. Informe del taller interregional de la OMS sobre el uso de la medicina tradicional en la atención primaria de salud. Ulaanbaatar (Mongolia); 2007.

2. OMS. Estrategia de la OMS sobre medicina tradicional 2002-2005. Ginebra: OMS; 2009.

3. Cardoso B, Porto, M. Experiencia Cubana en el estudio y aplicación de Medicamentos Herbarios. Rev. Cubana de Plant Med. La Habana, Cuba. 1997;2(1):30-34.

4. Almeida Vera L, Almeida Vera L. Fundamentación del modelo de gestión intercultural ecuatoriana en la atención primaria de salud. MEDISAN 2014;18(8):1210.

5. Jaimes-Roncancio MS, Betancur J, Cámara-Leret R. Palmas útiles en tres comunidades indígenas de La Pedrera, Amazonia colombiana. Caldasia [Internet]. 1 de enero de 2018;40(1):112-28. Disponible en: https://revistas.unal.edu.co/index.php/cal/article/view/68851

6. Soria N, Ramos P. Uso de plantas medicinales en la atención primaria de Salud en Paraguay: algunas consideraciones para su uso seguro y eficaz. Mem. Inst. Investig. Cienc. Salud 2015;3(2):8-17.

7. Cabrera L, Holmes W, McDaniel S. Flora del Paraguay. Compositae III Conservatoire et Jardin botanique de Geneve, Missouri Botanical Garden 1996; $349 \mathrm{p}$.

8. Cabrera L, Freire S. Flora del Paraguay. Compositae V. Conservatoire et Jardin botanique de Geneve. Missouri Botanical Garden; 1998. 212 p. 
9. Missouri Botanical Garden. Tropicos. 2021. Disponible en: https://www.tropicos.org

10. Instituto de Botánica Darwinion. Flora del CONOSUR. 2021. Disponible en: http://www.darwin.edu.ar/

11. Organización Panamericana de la Salud. CIE-10. Clasificación Estadística Internacional de Enfermedades y Problemas Relacionados con la Salud. 2013 [Accedido 15/07/2018]. 1158 p. Disponible en: http://iris.paho.org/xmlui/bitstream/handle/123456789/6282/Volum e1.pdf

12. Ortega-Cala L, Monroy-Ortiz C, Monroy-Martínez R, Colín-Bahena H, Flores-Franco G, Luna-Cavazos M, Monroy-Ortiz R. Plantas medicinales utilizadas para enfermedades del sistema digestivo en Tetela del Volcán, Estado de Morelos, México. Blacpma 2019; 18(2):106-129.

13.Zambrano-Intriago LF, Buenaño-Allauca MP, Mancera-Rodríguez NJ, Jiménez-Romero E. Estudio etnobotánico de plantas medicinales utilizadas por los habitantes del área rural de la Parroquia San Carlos, Quevedo, Ecuador. Rev. Univ. Salud 2015;17(1):97-111. Disponible en: 10.1186/1746-4269.

14. Godinez-Caraballo D, Volpato G. Plantas medicinales que se venden en el mercado de Río, Camagüey Cuba. Revista Mejicana de Biodiversidad 2008;79:243-259.

15. Dejani NN, Souza LC, Oliveira SRP, Neris DM, Rodolpho JMA, Correia RO, Rodrigues V, Sacramento LVS, Faccioli LH, Afonso A, Anibal FF. Immunological and parasitological parameters in Schistosoma mansoniinfected mice treated with crude extract. Immunobiol. 2014;219:627632.

16. Machado M, Sousa MC, Salgueiro L, Cavaleiro C. Effects of essential oils on the growth of Giardia lamblia trophozoites. Nat Prod Commun 2010; 5: 137-141.

17. Juárez-Vázquez MC, Carranza-Álvarez C, Alonzo-Castro AJ, GonzálezAlcaraz VF, Bravo-Acevedo E, Chamarro-Tinajero FJ, Solano E. Ethnobotany of medicinal plants in Xalpatlahuac, Guerrero, México. J Ethnopharmacol 2013; 148: 521-527.

18. Henao RSC, Martínez MJD, Pacheco GNL, Marín LJC. Actividad bactericida de extractos acuosos de Lippia alba (Mill.) N.E. Brown contra Helicobacter pylori. Rev. Col. Gastroenterol. 2011;26(2):82-87. Disponible en: http://www.scielo.org.co/pdf/rcg/v26n2/v26n2a02.pdf

19. Echemendía Salís CE, Morón Rodríguez FJ. Tintura de hojas de Psidium guajava L. en pacientes con diarrea aguda simple. Rev Cubana Plant Med [Internet]. 2004;9(3). Disponible en: http://scielo.sld.cu/scielo.php?script=sci_arttext\&pid=S102847962004000300008\&lng=es 
20. Fedorova VY. Agent for treating and preventing gastritis and gastric ulcer. RU Patent No 1904807. CAPLUS, 2015.

21. Castillo-Juárez I, González V, Jaime-Aguilar H, Martínez G, Linares E, Bye R, Romero I. Anti-Helicobacter pylori activity of plants used in Mexican traditional medicine for gastrointestinal disorders. J. Ethnopharmacol. 2009; 122: $402-405$.

22. De Farias Freire SM, Da Silva Emim JA, Lapa AJ, Souccar C, Brandao Torres LM. Analgesic and anti-inflammatory properties of Scoparia dulcis L. extracts and glutinol in rodents. Phytother Res. 1993;7(6):40814. 